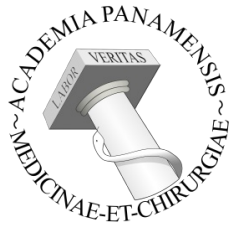

\title{
Artículos Científicos \\ Meningitis por Cryptococcus neoformans asociado a pacientes con VIH en Panamá. Cryptococcus neoformans meningitis related to HIV patients in Panama.
}

\author{
Villarreal Olmedo*, Cantón, Alfredo** \\ *Unidad de Enfermedades Infecciosas, Hospital Irma De Lourdes Tzanetatos, Panamá. **Servicio de Enfermedades \\ Infecciosas, Hospital Dr. Arnulfo Arias Madrid, Panamá.
}

Palabras claves: Cryptococcus neoformans, meningitis, VIH, SI$\mathrm{DA}$, anfotericina $\mathrm{B}$

Key words: Cryptococcus neoformans, meningitis, HIV, AIDS, Amphotericin B.

\section{Correspondencia:}

Villareal Olmedo

Correo electrónico:

villarreal.md@hotmail.com

Recibido: 11 de octubre de 2019 Aceptado: 18 de febrero de 2020 Publicado: 27 de marzo de 2020

Conflicto de interés: Los autores declaran no tener conflictos de intereses para la realización de este estudio. Este estudio fue aprobado por el Comité de Bioética de la Caja de Seguro Social.

\section{Resumen}

Introducción La meningitis criptocócica es una causa importante de mortalidad relacionado al SIDA en los países en vía de desarrollo, pudiendo ocasionar secuelas neurológicas, sobre todo en aquellos pacientes con recuentos de CD4 menor a $100 \mathrm{cel} /$ microlitro. Realizamos un estudio observacional, retrospectivo para evaluar la mortalidad, cuadro clínico, características citoquímicas y microbiológicas del líquido cefalorraquídeo, así como la respuesta al tratamiento de los pacientes con meningitis criptocócica y VIH. Material y método Realizamos un estudio observacional, retrospectivo para evaluar la mortalidad, cuadro clínico, características citoquímicas y microbiológicas del líquido cefalorraquídeo, así como la respuesta al tratamiento de los pacientes con meningitis criptocócica y VIH. que fueron admitidos en sala de Infectologia del Complejo Hospitalario Dr. Arnulfo Arias Madrid entre enero del 2013 a diciembre del 2015. Los datos recogidos de los expedientes, se anotaron en el instrumento de recolección para posteriormente crear una matriz de datos en Excel y posterior análisis con el programa estadístico IBM SPSS statistics data editor 23.

Resultados De un total de 29 pacientes, 17 cumplieron los requisitos de inclusión: todos eran $\mathrm{VIH}$ positivo, de los cuales el $85 \%$ eran masculino, con edad promedio de 38 años y conteo de linfocitos T CD4 en $47 \mathrm{cel} /$ microlitro (Tabla 1). El 58.8\% (10/17) de los pacientes se presentaron sin alteración en el estado de consciencia o rigidez nucal (Figura 1). Fiebre $52.9 \%$ (9/17) de los casos El síntoma capital fue la cefalea, en 16 de los 17 casos. La mediana en la presión de apertura fue de $31 \mathrm{~cm} \mathrm{H}_{2} \mathrm{O}\left(12\right.$ y $\left.54 \mathrm{~cm} \mathrm{H}_{2} \mathrm{O}\right)$, con glóbulos blancos en $31 \mathrm{cel} / \mathrm{mm} 3\left(0\right.$ a $\left.660 \mathrm{cel} / \mathrm{mm}^{3}\right)$, glucosa $28.5 \mathrm{mg} / \mathrm{dl}$ (2 y $55 \mathrm{mg} /$ dl) y proteínas en $124 \mathrm{~g} / \mathrm{L}$ (33 a $401 \mathrm{~g} / \mathrm{L}$ ) (Figura 2). Todas las muestras de líquido cefalorraquídeo presentaban levadura encapsulada en la tinción con tinta china y se aisló Cryptococcus neoformans. El $70.6 \%$ de los pacientes recibieron anfotericina B deoxicolato más fluconazol. La mortalidad intrahospitalaria fue de $5.8 \%(1 / 17)$ con un promedio de 16 días de hospitalización (Tabla 1). Conclusión La cefalea fue el principal síntoma. Sin embargo, más de la mitad de nuestros pacientes no presentaron fiebre, signos meníngeos ni alteraciones neurológicas. La presión de apertura elevada es un hallazgo común, así como la pleocitosis con proteinorraquia. En los pacientes con VIH la terapia antirretroviral y los esquemas de anfotericina más fluconazol a dosis altas han logrado disminuir la mortalidad; sin embargo, en Panamá no contamos con flucitosina que es la primera línea de tratamiento, que podría disminuir aún más la mortalidad. Se requieren pruebas de detección temprana para criptococo y así disminuir la morbimortalidad de estos pacientes.

\begin{abstract}
Introduction Cryptococcal meningitis is an important cause of AIDS-related mortality in developing countries, and can cause neurological sequelae, especially in patients with CD4 counts less than $100 \mathrm{cel} /$ microliter. We performed an observational, retrospective study to assess mortality, clinical picture, cytochemical and microbiological characteristics of cerebrospinal fluid, as well as the response to treatment of patients with cryptococcal meningitis and HIV. Material and methods We performed an observational,
\end{abstract}

Órgano oficial de la Academia Panameña de Medicina y Cirugía y del Instituto Commemorativo Gorgas para Estudios de la Salud (ICGES). Indexado en LILACS Y EBSCO.

http://dx.doi.org/10.37980/im.journal.rmdp.2020848
R M P

2020: Volumen 40(1): 21-24 
retrospective study to assess mortality, clinical picture, cytochemical and microbiological characteristics of cerebrospinal fluid, as well as the response to treatment of patients with cryptococcal meningitis and HIV. who were admitted to the Infectology room of the Dr. Arnulfo Arias Madrid Hospital Complex between January 2013 to December 2015. The data collected from the files was recorded in the collection instrument to later create a data matrix in Excel and subsequent analysis with the IBM SPSS statistics data editor 23. Results Of a total of 29 patients, 17 met the inclusion requirements: all were HIV positive, of which $85 \%$ were male, with an average age of 38 years and CD4 T lymphocyte count at 47 cell / microliter (Table 1). 58.8\% (10/17) of the patients presented without alteration in the state of consciousness or nuchal rigidity (Figure 1). Fever $52.9 \%(9 / 17)$ of the cases the main symptom was headache, in 16 of the 17 cases. The median opening pressure was $31 \mathrm{cmH}_{2} \mathrm{O}\left(12\right.$ and $\left.54 \mathrm{cmH}_{2} \mathrm{O}\right)$, with white blood cells at $31 \mathrm{cel} / \mathrm{mm} 3$ (0 to $660 \mathrm{cel} / \mathrm{mm}^{3}$ ), glucose $28.5 \mathrm{mg} / \mathrm{dl}(2$ and $55 \mathrm{mg} / \mathrm{dl}$ ) and protein. at $124 \mathrm{~g} / \mathrm{L}$ (33 to $401 \mathrm{~g} / \mathrm{L}$ ) (Figure 2). All cerebrospinal fluid samples had yeast encapsulated in Indian ink staining and Cryptococcus neoformans was isolated. $70.6 \%$ of patients received amphotericin B deoxycholate plus fluconazole. In-hospital mortality was $5.8 \%$ (1/17) with an average of 16 days of hospitalization (Table 1). Conclusion Headache was the main symptom; however, more than half of our patients had no fever, meningeal signs, or neurological abnormalities. High opening pressure is a common finding, as is pleocytosis with proteinorrachy. In HIV patients, antiretroviral therapy and amphotericin plus fluconazole regimens at high doses have reduced mortality, however, in Panama we do not have flucytosine, which is the first line of treatment, which could further decrease mortality. Early detection tests are required for Cryptococcus and thus decrease the morbidity and mortality of these patients.

\section{INTRODUCCIÓN}

El criptococo es un hongo, que comúnmente vive en la tierra. Puede entrar en el cuerpo cuando respira el polvo o el excremento seco de pájaros [1]. No parece transmitirse de persona a persona [2]. La meningitis criptocócica es una causa importante de mortalidad relacionada al SIDA en los países en vía de desarrollo, pudiendo ocasionar secuelas neurológicas, sobre todo en aquellos pacientes con recuentos de CD4 menor a $100 \mathrm{cel} / \mathrm{microli}-$ tro [2]. Para el año 2000 se presentaban en Panamá 20 casos por año. Con la introducción de la triple terapia, hubo una disminución a 4 casos por año. Sin embargo, en los últimos 5 años se registran en promedio 6 casos por año, con una mortalidad que supera el $50 \%[1,3,4]$. Los primeros síntomas de meningitis incluyen fiebre, fatiga, rigidez nucal, dolor de cabeza, náuseas y vómitos, confusión, visión borrosa o fotofobia a luz intensa. Los síntomas pueden aparecer progresivamente [5,6]. Con este estudio buscamos analizar en nuestra población de pacientes con VIH, las características clínico epidemiológicas, características barométricas y citoquimicas del líquido cefalorraquídeo, así como la mortalidad con los, esquemas de antifúngicos empleados en la actualidad $[7,8,9]$. Un diagnóstico temprano pudiera repercutir en las secuelas y mortalidad de estos pacientes, por lo que se debe realizar antígeno de criptococo en el suero de los pacientes asintomáticos con niveles de CD4 menor de $100 \mathrm{cel} /$ microlitro [10].

\section{MATERIALES Y MÉTODOS}

Realizamos un estudio observacional, retrospectivo para evaluar la mortalidad, cuadro clínico, características cito- químicas y microbiológicas del líquido cefalorraquídeo así como la respuesta al tratamiento de los pacientes con meningitis criptocócica y VIH.

Se revisaron los expedientes clínicos de aquellos pacientes con diagnóstico de VIH y meningitis por Cryptococcus, que fueron admitidos en sala de Infectologia del Complejo Hospitalario Dr. Arnulfo Arias Madrid entre enero del 2013 a diciembre del 2015; todos los pacientes eran mayor de 18 años, con cultivos de líquido cefalorraquídeo positivo para criptococos neoformans. No fueron incluidos aquellos pacientes que fueron admitidos a unidad de cuidados intensivos, que, a pesar de encontrar levadura encapsulada en la tinta china, tenían cultivos negativos en líquido cefalorraquídeo y aquellos que fallecieron en las primeras 24 horas de la hospitalización y no tenían los estudios diagnósticos definitivos. Los datos recogidos de los expedientes, previa aprobación por el jefe de servicio y comité de ética, se anotaron en el instrumento de recolección para posteriormente crear una matriz de datos en Excel y posterior análisis con el programa estadístico IBM SPSS statistics data editor 23.

\section{RESULTADOS}

De un total de 29 pacientes, 17 cumplieron los requisitos de inclusión: todos eran VIH positivo, de los cuales el $85 \%$ eran masculino, con edad promedio de 38 años y conteo de linfocitos T CD4 en $47 \mathrm{cel} /$ microlitro (Tabla 1). El 58.8\% (10/17) de los pacientes se presentaron sin alteración en el estado de consciencia o rigidez nucal (Figura 1). Fiebre $52.9 \%$ (9/17) de los casos. El síntoma capital fue la cefalea, en 16 de los 17 casos. La mediana en la presión de apertura fue de $31 \mathrm{~cm} \mathrm{H} 2 \mathrm{O}$ (12 y $54 \mathrm{~cm}$ $\mathrm{H} 2 \mathrm{O}$ ), con glóbulos blancos en $31 \mathrm{cel} / \mathrm{mm}^{3}$ ( 0 a $660 \mathrm{cel} /$ 
Tabla № 1. Distribución de los pacientes según sexo, edad, conteo de CD4, días de hospitalización, tratamiento antirretroviral, antimicótico y las características neurológicas al momento de su ingreso

\begin{tabular}{|c|c|}
\hline \multicolumn{2}{|c|}{ CARACTERISTICAS } \\
\hline Sexo & frecuencia/\% \\
\hline Masculino & $15(88.2)$ \\
\hline Femenino & $2(11.8)$ \\
\hline Edad & años \\
\hline Mediana & 38 \\
\hline Rango & $26-57$ \\
\hline Conteo de CD4 & por $\mathrm{mm} 3$ \\
\hline Mediana & 47 \\
\hline Rango & $12-276$ \\
\hline Terapia antirretroviral & frecuencia/\% \\
\hline Sin tratamiento & $2(11.8)$ \\
\hline Con tratamiento & $15(88.2)$ \\
\hline FTC/EFV/TDF & $10(58.8)$ \\
\hline Otros esquemas & $5(29.4)$ \\
\hline Tratamiento antimicótico & frecuencia/ $\%$ \\
\hline Anfo B + Fluconazol IV 800 mg & $12(70.6)$ \\
\hline Anfo B & $2(11.8)$ \\
\hline Otros & $3(17.7)$ \\
\hline Alteraciones Neurológicas & $\%$ \\
\hline Con alteraciones & $7(41.2)$ \\
\hline Sin alteraciones & $10(58.8)$ \\
\hline Hospitalización & dlas \\
\hline Mediana & 16 \\
\hline Rango & Jun-47 \\
\hline
\end{tabular}

Fuente: Base de datos. Registros médicos del Complejo Hospitalario Dr. Arnulfo Arias Madrid. Panamá 2017

$\mathrm{mm}^{3}$ ), glucosa $28.5 \mathrm{mg} / \mathrm{dl}$ (2 y $55 \mathrm{mg} / \mathrm{dl}$ ) y proteínas en $124 \mathrm{~g} / \mathrm{L}$ (33 a $401 \mathrm{~g} / \mathrm{L}$ ) (Figura 2). Todas las muestras de líquido cefalorraquídeo presentaban levadura encapsulada en la tinción con tinta china y se aisló Cryptococcus neoformans. El $70.6 \%$ de los pacientes recibieron anfotericina $\mathrm{B}$ deoxicolato más fluconazol. La mortalidad intrahospitalaria fue de $5.8 \%(1 / 17)$ con un promedio de 16 días de hospitalización (Tabla 1).

\section{DISCUSIÓN}

De un total de 29 pacientes diagnosticados con criptococosis meníngea en la sala de Infectología del C.H.Dr.A.A.M. entre enero del 2013 y diciembre del 2015, se logró revisar los expedientes clínicos de 17 pacientes que cumplían con los criterios de inclusión. Del total de los pacientes el sexo masculino fue el predominante con $88.2 \%(15)$ y solo $11.8 \%$ (2) de los pacientes fueron del
Figura 1. Distribución de los pacientes de acuerdo con os síntomas neurológicos que presentaron a su ingreso

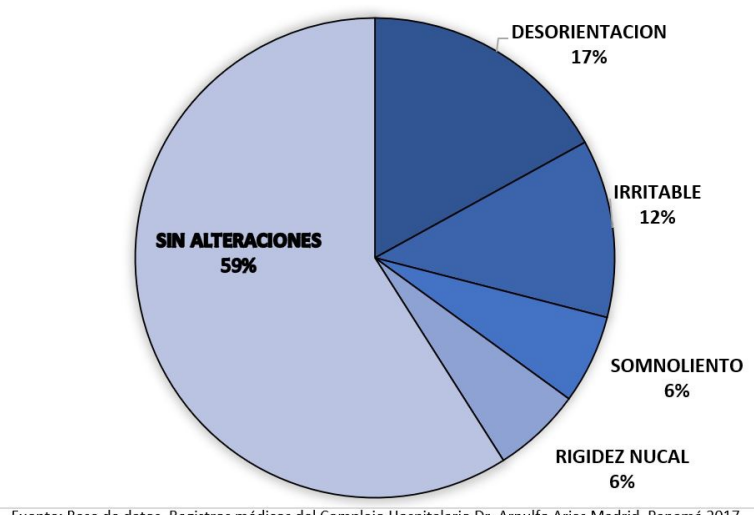

Fuente: Base de datos. Registros médicos del Complejo Hospitalario Dr. Arnulfo Arias Madrid. Panamá 2017

Figura 2. Características barométricas y citoquimicas de las punciones lumbares

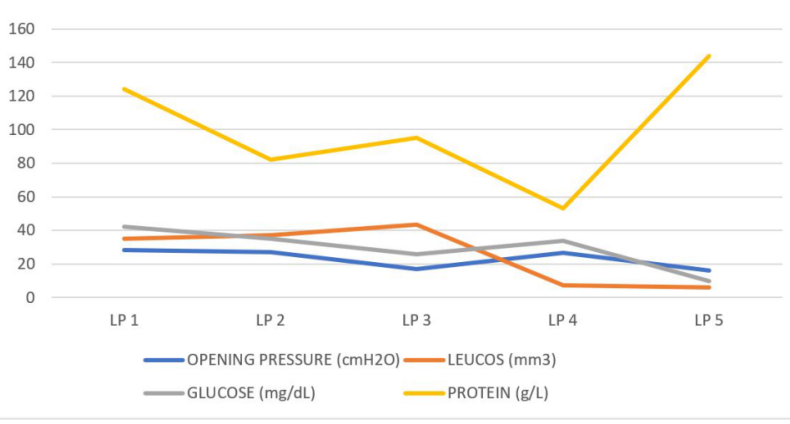

Fuente: Base de datos. Registros médicos del Complejo Hospitalario Dr. Arnulfo Arias Madrid. Panamá 2017

sexo femenino; continúa siendo la población masculina la más afectada al compararlo con serie de casos previas en Panamá, donde se reportaron $60.7 \%$ de hombres vs $39.3 \%$ de mujeres (Sierra et al., 1996), series de casos en América Latina reportaron 77\% (Sierra Saldívar, 2013) y 75\% (Ramírez et al., 2015). El rango de edad fue entre 26 y 57 años, similar a los reportados a nivel mundial y similar al reportado por Sierra et al en el Hospital Santo Tomas en Panamá (23-53 años). la mediana de linfocitos T CD4 fue de $47 \mathrm{cel} / \mathrm{mm}^{3}$, inmunodepresión severa, lo esperado si tomamos en cuenta que la meningitis por criptococo se presenta con niveles menor a $100 \mathrm{cel} / \mathrm{mm}^{3}$. El síntoma inicial predominante fue la cefalea con $94.1 \%$ (16) de los casos y la fiebre en poco más de la mitad de ellos $52.9 \%$ (9); similar a lo reportado por Sierra et al donde el síntoma capital fue la cefalea y los vómitos. A diferencia de las series previas, nuestros pacientes en su mayoría no presentaban signos ni síntomas neurológicos, ni tampoco presentaron alteración en la escala de Glasgow al momento del diagnóstico. La presión de apertura inicial en la punción lumbar es elevada, encontramos una mediana de 58.5 $\mathrm{cm} \mathrm{H} 2 \mathrm{O}$, en este estudio incluimos las presiones de apertura sucesivas, datos no reportados en las series de casos. El aumento de la presión de apertura es un hallazgo común, pero no patognomónico, así como la pleocitosis con un aumento de proteínas en el líquido cefalorraquídeo. 
En pacientes con $\mathrm{VIH}$ que reciben terapia antirretroviral y anfotericina más fluconazol en dosis altas, hemos podido reducir la mortalidad, sin embargo, en Panamá no tenemos flucitosina, que es la primera línea de tratamiento, que podría reducir aún más la mortalidad. En Panamá todavía vemos pacientes que llegan en fase de SIDA, con diagnósticos tardíos, por lo que son necesarias pruebas de detección temprana para el criptococo y, por lo tanto, reducen la morbilidad y la mortalidad de estos pacientes.

\section{CONCLUSIÓN}

Las características clínicas de los pacientes evaluados son similares a las reportadas en estudios previos tanto locales como internacionales, siendo la cefalea el principal síntoma; sin embargo, más de la mitad de nuestros pacientes no presentaron fiebre, signos meníngeos ni alteraciones neurológicas. La presión de apertura elevada es un hallazgo común, así como la pleocitosis con proteinorraquia. En los pacientes con VIH la terapia antirretroviral y los esquemas de anfotericina más fluconazol a dosis altas han logrado disminuir la mortalidad, sin embargo, en Panamá no contamos con flucitosina que es la primera línea de tratamiento, que podría disminuir aún más la mortalidad. Se requieren pruebas de detección temprana para criptococo y así disminuir la morbimortalidad de estos pacientes.

\section{REFERENCIAS}

[1] Jarvis, J. N., et al. (2014). Determinants of mortality in a combined cohort of 501 patients with HIV-associated Cryptococci meningitis: Implications for improving outcomes. Clinical Infectious Diseases, 58(5), 736-745.
[2] Jonathan E. Kaplan, et al. (2009). Guidelines for Prevention and Treatment of Opportunistic Infections in HIV-Infected Adults and Adolescents. The HIV Medicine Association of the Infectious Diseases Society of America, 1(198), 1.

[3] González, C., et al. (2013). Revista Médica de Panamá Meningitis por Cryptococcus neoformans en pacientes con SIDA, 3(2), 3-7.

[4] Sierra, L. O., et al. (1996). [Cryptococcus neoformans meningitis in patients with AIDS at the Saint Thomas Hospital]. Revista Médica de Panamá, 21, 46-50.

[5] Ramírez, S., et al. (2015). Criptococosis cerebral: descripción de una serie de casos con presentaciones típicas y atípicas en el Hospital Universitario San José Infantil de Bogotá Cerebral cryptococcosis: description of a series of cases of atypical clinical presentation, 31(2), 158-166.

[6] Brizendine, K. D., et al. (2013). Predictors of Mortality and Differences in Clinical Features, among $\mathrm{Pa}$ tients with Cryptococcosis According to Immune Status. PLoS ONE, 8(3).

[7] Day, J. N., et al. (2013). Combination Antifungal Therapy for Cryptococcal Meningitis. New England Journal of Medicine, 368(14), 1291-1302

[8] Yao, Z. W., et al. (2014). Comparison of flucytosine and fluconazole combined with amphotericin B for the treatment of HIV-associated cryptococcal meningitis: A systematic review and meta-analysis. European Journal of Clinical Microbiology and Infectious Diseases, 33(8), 1339-1344

[9] Merry, M., et al. (2016). Cryptococcal Meningitis Treatment Strategies Affected by the Explosive Cost of Flucytosine in the United States: A Cost-effectiveness Analysis. Clinical Infectious Diseases, 62(12), 1564-1568.

[10] Perfect, J. R., et al. (2015). Cryptococcosis diagnosis and treatment: What do we know now? Fungal Genetics and Biology, 78, 49-54. 